\title{
A Case of Polyautoimmunity: Celiac Hepatitis, Grave's Disease and Autoimmune Hemolytic Anemia
}

\author{
Nazish Butt1, Muhammad Ali Khan1, Zain Abid2 and Farhan Haleem1 \\ ${ }^{1}$ Gastroenterology Section, Ward 23 / 2Oncology Section, Ward 4, Jinnah Postgraduate Medical Centre, Karachi, Pakistan
}

\begin{abstract}
Celiac disease $(C D)$ is an autoimmune disorder with high incidence of multi organ involvement; especially, gastrointestinal manifestations and an increased risk of malignancies. Here we report a case of CD with celiac hepatitis, autoimmune hemolytic anemia (AIHA) and Grave's disease (GD) with their complications.

Polyautoimmunity requires comprehensive analysis. While CD and GD were previously diagnosed, AlHA and cirrhosis were diagnosed during admission upon extensive work-up. Similarly, other autoimmune etiologies, such as autoimmune hepatitis (AlH), and/or primary biliary cholangitis were ruled out.

All three diseases were treated afresh with strict adherence to a gluten-free diet (GFD) and carbimazole along with addition of medications for cirrhosis complicated by ascites. This was a rare case where non-adherence to a GFD led to such severe adverse events. A case of celiac hepatitis presenting with such a wide array of signs and symptoms has rarely been reported in the literature and the management of this patient was unique and challenging.
\end{abstract}

Key Words: Celiac hepatitis, Grave's disease, Autoimmune hemolytic anemia, Polyautoimmunity.

How to cite this article: Butt N, Khan MA, Abid Z, Haleem F. A case of polyautoimmunity: celiac hepatitis, Grave's disease and autoimmune hemolytic anemia. J Coll Physicians Surg Pak 2019; 29 (Supplement 2):S106-S108.

\section{INTRODUCTION}

Celiac disease (CD) is defined as a small bowel disorder characterised by mucosal inflammation, villous atrophy and crypt hyperplasia, which occurs on exposure to dietary gluten and demonstrates improvement after withdrawal of gluten from the diet. ${ }^{1}$

The gold standard for the diagnosis of CD is duodenal biopsy that demonstrates villous atrophy of variable grades. ${ }^{2}$ It is the most common food intolerance in the world and can present at any age with gastrointestinal symptoms such as chronic diarrhea, weight loss, vomiting, flatulence, and abdominal distention; however, extra-intestinal manifestations are not uncommon. ${ }^{3}$ The common extra-intestinal manifestations include thyroid disorders 4 , autoimmune hepatitis $(\mathrm{AlH})^{5}$, hematological disorders $^{6}$, vitiligo and others.

Polyautoimmunity is defined as the presence of two or more autoimmune diseases (AD) in a single human being. ${ }^{7}$ When three or more ADs coexist, this is known as multiple autoimmune syndrome (MAS). ${ }^{8} \mathrm{CD}$, Grave's disease (GD) and autoimmune hemolytic anemia (AIHA) are known to be a part of the spectrum that is polyautoimmunity; although, each may present as a singular

Correspondence to: Dr. Muhammad Ali Khan, Section of Gastroenterology, Ward No. 23, Jinnah Postgraduate Medical

Centre, Karachi, Pakistan

E-mail: aleekhan3333@gmail.com

Received: December 12, 2018; Revised: June 22, 2019;

Accepted: August 22, 2019 disorder as well. No temporal relationship has been established between different autoimmune processes in patients with polyautoimmunity.

\section{CASE REPORT}

A 19-year female, diagnosed case of CD for eight years and GD for five years, presented at the Emergency Department with history of persistent jaundice for two months. There was also history of chronic diarrhea, fatigue and shortness of breath on exertion. She was receiving carbimazole, $10 \mathrm{mg}$, BID, and was admittedly non-compliant to both a gluten-free diet (GFD) and the medications. She had completely stopped taking the medications for six weeks at the time of presentation.

On examination, the patient was hemodynamically stable, jaundiced and anemic. She had thin coarse hair, fine tremor, exophthalmos (Figure 1), a positive lid lag, Von Graefe's and lid retraction signs. Fluid thrill and shifting dullness were also present. There were also patches of hypo-pigmented skin in the hands, forearms and legs, consistent with vitiligo.

Initial laboratory tests showed hemoglobin $(\mathrm{Hb})$ of $8.8 \mathrm{~g} / \mathrm{dL}$, mean corpuscular volume (MCV), $85.5 \mathrm{fL}$, platelets, $68 \times 109 / \mathrm{L}$, and white blood cell count (WBC) of $9.2 \times 109 / \mathrm{L}$. Total bilirubin was $11.30 \mathrm{mg} / \mathrm{dl}$, direct bilirubin, 5.40 $\mathrm{mg} / \mathrm{dl}$, alanine aminotransferase (ALT), $145 \mathrm{U} / \mathrm{L}(0-41 \mathrm{U} / \mathrm{L})$, aspartate transaminase (AST) $55 \mathrm{U} / \mathrm{L}(0-43 \mathrm{U} / \mathrm{L})$, gamma-glutamyl transferase (GGT), $33 \mathrm{U} / \mathrm{L}$ (0-31 U/L), alkaline phosphatase, $142 \mathrm{U} / \mathrm{L}(65-306 \mathrm{U} / \mathrm{L})$, albumin, $2.2 \mathrm{~g} / \mathrm{dl}$, international normalised ratio (INR) 1.12, urea, $17 \mathrm{mg} / \mathrm{dl}$ and creatinine, $0.6 \mathrm{mg} / \mathrm{dl}$. 


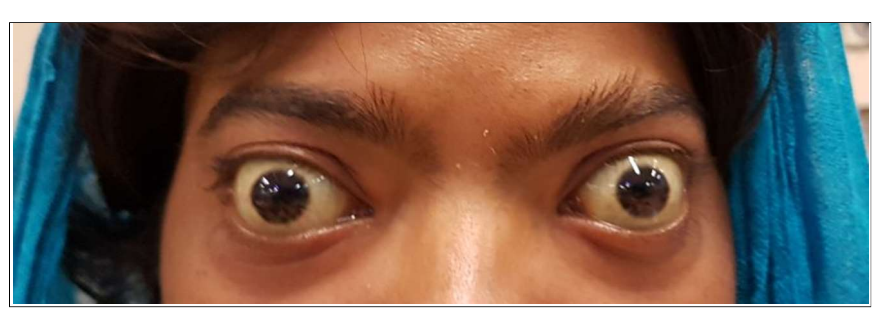

Figure 1: Bilateral exophthalmos seen due to Grave's disease.

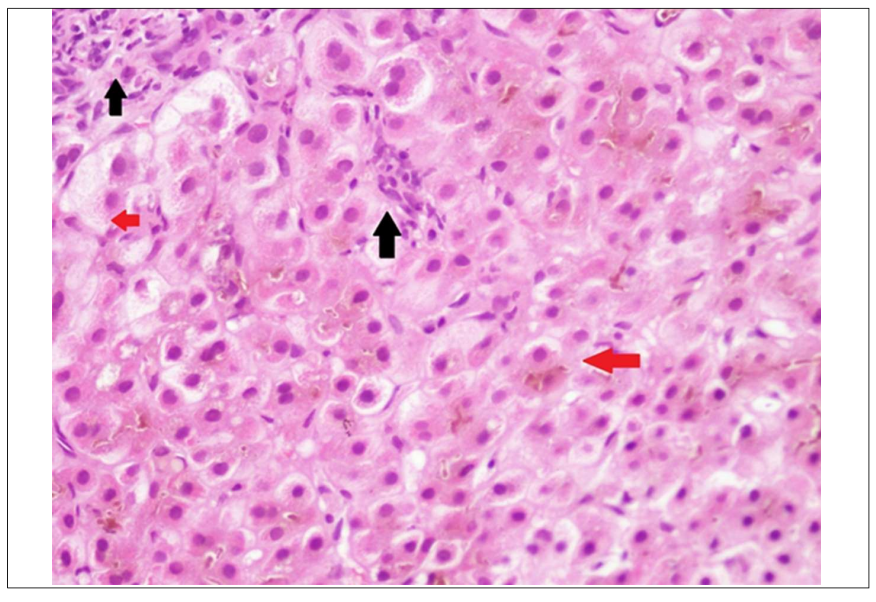

Figure 2: Histopathology slide: Balloon degeneration of hepatocytes with damage and extensive areas of cholestasis (red arrows). Moderate to severe inflammation is present comprising of neutrophils, plasma cells and lymphocytes (black arrows)

A raised lactate dehydrogenase $(\mathrm{LDH})$ of $644 \mathrm{U} / \mathrm{L}(100-$ $190 \mathrm{U} / \mathrm{L}$ ) with a corrected reticulocyte count of $3.91 \%$ raised the possibility of AIHA. Indirect and direct Coomb's tests were both positive. These results were consistent with AlHA. Blood culture was done after taking a hematological consult, it was unremarkable. Chest X-ray (PA) view was normal.

Serum electrolytes were normal. Complete thyroid profile was consistent with hyperthyroidism (Free T3, $2.63 \mathrm{ng} / \mathrm{mL}$, Free T4, $8.53 \mathrm{pg} / \mathrm{dl}, \mathrm{TSH}, 0.01 \mathrm{ulU} / \mathrm{mL})$. Esophagogastroduodenoscopy (EGD) showed fissuring and scalloping of the duodenal folds consistent with CD.

Viral serologies for hepatitis A, B, C and E were negative. The initial laboratory data raised the possibility of an underlying autoimmune disorder associated with $C D$ and the Anti-nuclear antibodies (ANA) profile including anti-smooth muscle antibody (ASMA) with liver kidney microsome type 1 antibody (anti-LKM1) were performed; which were negative. Further testing with immunoglobulin IgG levels was unremarkable as well, effectively ruling out autoimmune hepatitis (AlH).

The liver biopsy (Figure 2) showed cholestasis and bile ductular damage and overlap with conditions such as primary biliary cholangitis; and/or primary sclreosing Cholangitis could not be ruled out. Therefore, a magnetic resonance cholangiopancreatography (MRCP) was performed to assess the disease status; the results indicated development of moderate ascites and cirrhosis.
The patient was subsequently put on a strict GFD; carbimazole was increased to $20 \mathrm{mg}$, BID, after an endocrinological review. The patient responded well to these measures, the diarrhea subsided, and the liver enzymes started to show a resolving pattern.

In a multidisciplinary meeting, the case was discussed and a diagnosis of celiac hepatitis was agreed upon. On follow-up, the patient has been compliant to medications for decompensated liver disease and a GFD, which resulted in resolution of most gastrointestinal (GI) symptoms. Symptoms of hyperthyroidism have also improved with carbimazole.

However, anemia and thrombocytopenia persisted in the light of the patient's decompensated liver state. Ascites responded to diuretics. There have been no episodes of encephalopathy and hemetemesis. The liver enzymes have resolved over time to their normal ranges. Due to the advanced nature of liver disease, steroids were withheld; steroids would have resulted in further deteriotion of ascites and liver function.

\section{DISCUSSION}

The association between $C D$ and liver disease is widely recognised in the pediatric population. The spectrum of celiac hepatitis may range from unexplained transaminasaemia to cirrhosis. ${ }^{9}$ Similarly, CD must be considered a differential in cases of cryptogenic persistent hypertransaminasemia. Studies have reported that transaminases are elevated in $39-47 \%$ of celiac adults and in $26-57 \%$ of children at diagnosis of CD.

Liver biopsy demonstrates histological changes such as Kupffer cell hyperplasia, mononuclear cell infiltration, steatosis, and mild fibrosis. A high number of patients demonstrate reduction of transaminases to normal levels after initiating a GFD.

The so-called celiac hepatitis is a frequent, benign and clinically silent condition, which resolves on a GFD. This case highlights the importance of compliance to GFD and the consequences of refusal to do so.

The prevalence of autoimmune thyroid disease is significantly increased in patients with $C D$ when compared to the general population and ranges between 2 to $7 \%$. However this prevalence is even higher $(26.2 \%)$ in the pediatric population; even in patients on GFD, the prevalence of autoimmune thyroid disease in this group is $10 \%$. Females are more predisposed.

In particular, HLA DQ2 haplotype and a positive family history of autoimmune disorders seem to favour development of GD with CD. Up to $14 \%$ and $2 \%$ of patients with GD have antibodies to gliadin (AGA) and tissue transglutaminase (anti-tTG), respectively; thus routine screening should be considered in all patients with $C D$.

There are no definitive guidelines on management of GD with $C D$. There is also lack of data regarding response 
to a GFD and progression of the disease. Thus, the management remains delphic, although, conventional wisdom would be to treat each condition separately.

AlHA has been reported with both $C D$, and more commonly with GD. There have been reports of AlHA with CD as early as $1984^{10}$ and the first reported case of CD with Evan's syndrome was reported in 2007. But, this case of CD with both GD and AIHA is the first to be reported in the literature to the best of our knowledge.

Nearly $60 \%$ CD-associated susceptibility loci can be found in other autoimmune conditions, suggesting similar pathogenic mechanisms. This is most clearly evident in the association between $C D$ and diabetes mellitus, type I. Presence of $C D$ in autoimmune polyglandular syndrome (APS) has also been reported and is yet another example of polyautoimmunity associated with $C D$.

AlH and drug-induced liver injury (DILI) were differentials in our case. AlH was ruled out on the basis of laboratory and histological analyses. As for DILI, no temporal relationship could be ascertained between drug (carbimazole) intake and onset of symptoms prior to presentation. However, at one year follow-up, the liver enzymes showed improvement despite continued intake of carbimazole, making DILI highly unlikely.

In conclusion, this is a rare case of multiple autoimmune pathologies afflicting the patient with $\mathrm{CD}$, presenting with different signs and symptoms specific for each disease and possible overlapping underlying mechanisms, augmented by non-adherence to a GFD. The exact role of gluten in the pathogenesis of extra-intestinal autoimmunity has not been fully understood yet.

\section{PATIENT'S CONSENT:}

Informed consent was taken in all matters. Patient confidentiality was made certain. A parent was always present to see the overall process.

\section{CONFLICT OF INTEREST:}

Authors declared no conflict of interest.

AUTHORS' CONTRIBUTION:

NB: Supervisor, author, data collect.

MAK: Author, data collect.

ZA: Data collect.

$\mathrm{FH}$ : Data collect.

\section{REFERENCES}

1. Kelly CP, Bai JC, Liu E, Leffler DA. Gastroenterology 2015; 148:1175-86.

2. Ciaccio EJ, Bhagat G, Tennyson CA, Lewis SK, Hernandez L, Green PH. Quantitative assessment of endoscopic images for degree of villous atrophy in celiac disease. Dig Dis Sci 2011 Mar; 56:805-11.

3. Guandalini S, Assiri A. Celiac disease: A review. JAMA Pediatr 2014; 168:272-8.

4. Hadithi M, de Boer H, Meijer JWR. Coeliac disease in Dutch patients with Hashimoto's thyroiditis and vice versa. World $J$ Gastroenterol 2007; 13:1715-22.

5. Vajro P, Vajro P, Paolella G, Maggiore G, Giordano G. Pediatric celiac disease, cryptogenic hypertransaminasemia, and autoimmune hepatitis. J Pediatr Gastroenterol Nutr 2013; 56: 663-70.

6. Stenhammar L, Ljunggren CG. Thrombocytopenic purpura and coeliac disease. Acta Paediatr Scand 1988; 77:764-6.

7. Bliddal $\mathrm{S}$, Nielsen $\mathrm{CH}$, Feldt-Rasmussen U. Recent advances in understanding autoimmune thyroid disease: The tallest tree in the forest of polyautoimmunity. F1000 Res $2017 ; 6: 1776$.

8. Anaya JM. The diagnosis and clinical significance of polyautoimmunity. Autoimmun Rev 2014; 13:423-6.

9. Majumdar K, Sakhuja P, Puri AS, Gaur K, Haider A, Gondal R. Coeliac disease and the liver: Spectrum of liver histology, serology and treatment response at a tertiary referral centre. J Clin Pathol 2017; 29.

10. Miller DG. Coeliac disease with autoimmune haemolytic anaemia. Postgrad Med J 1984; 60:629-30. 